\title{
Estimation of Acute Infarct Volume with Reference Maps: A Simple Visual Tool for Decision Making in Thrombectomy Cases
}

\author{
Dong-Eog Kim, ${ }^{\mathrm{a}, *}$ Wi-Sun Ryu, ${ }^{\mathrm{a}, *}$ Dawid Schellingerhout, ${ }^{\mathrm{b}}$ Han-Gil Jeong, ${ }^{\mathrm{c}}$ Paul Kim, ${ }^{\mathrm{a}}$ Sang-Wuk Jeong, \\ Man-Seok Park, ${ }^{\mathrm{d}}$ Kang-Ho Choi, ${ }^{\mathrm{d}}$ Joon-Tae Kim, ${ }^{\mathrm{d}}$ Beom Joon Kim, ${ }^{\mathrm{c}}$ Moon-Ku Han, ${ }^{\mathrm{c}}$ Jun Lee, ${ }^{\mathrm{e}}$ \\ Jae-Kwan Cha, ${ }^{\mathrm{f}}$ Dae-Hyun Kim, ${ }^{\mathrm{f}}$ Hyun-Wook Nah, ${ }^{\mathrm{f}}$ Soo Joo Lee, ${ }^{\mathrm{g}}$ Jae Guk Kim, ${ }^{\mathrm{g}}$ Keun-Sik Hong, ${ }^{\mathrm{h}}$ \\ Yong-Jin Cho, ${ }^{\mathrm{h}}$ Hong-Kyun Park, ${ }^{\mathrm{h}}$ Byung-Chul Lee, ${ }^{\mathrm{i}}$ Kyung-Ho Yu, ${ }^{\mathrm{i}}$ Mi-Sun Oh, ${ }^{\mathrm{i}}$ Jong-Moo Park, ${ }^{\mathrm{j}}$ \\ Kyusik Kang, ${ }^{\mathrm{j}}$ Kyung Bok Lee, ${ }^{\mathrm{k}}$ Tai Hwan Park, ${ }^{\mathrm{l}}$ Sang-Soon Park, ${ }^{\mathrm{l}}$ Yong-Seok Lee, ${ }^{\mathrm{m}}$ Hee-Joon Bae ${ }^{\mathrm{c}}$ \\ aKorean Brain MRI Data Center, Dongguk University Ilsan Hospital, Dongguk University College of Medicine, Goyang, Korea \\ ${ }^{b}$ Departments of Radiology and Cancer Systems Imaging, University of Texas M. D. Anderson Cancer Center, Houston, TX, USA \\ 'Department of Neurology, Seoul National University Bundang Hospital, Seoul National University College of Medicine, Seongnam, Korea \\ ${ }^{d}$ Department of Neurology, Chonnam National University Hospital, Chonnam National University Medical School, Gwangju, Korea \\ eDepartment of Neurology, Yeungnam University Hospital, Yeungnam University College of Medicine, Daegu, Korea \\ fDepartment of Neurology, Dong-A University Hospital, Dong-A University College of Medicine, Busan, Korea \\ ${ }^{9}$ Department of Neurology, Eulji University Hospital, Eulji University, Daejeon, Korea \\ hDepartment of Neurology, Inje University Ilsan Paik Hospital, Inje University College of Medicine, Goyang, Korea \\ 'Department of Neurology, Hallym University Sacred Heart Hospital, Hallym University College of Medicine, Anyang, Korea \\ 'Department of Neurology, Nowon Eulji Medical Center, Eulji University School of Medicine, Seoul, Korea \\ kDepartment of Neurology, Soonchunhyang University Seoul Hospital, Soonchunhyang University College of Medicine, Seoul, Korea \\ 'Department of Neurology, Seoul Medical Center, Seoul, Korea \\ mDepartment of Neurology, Seoul Metropolitan Government Seoul National University Boramae Medical Center, Seoul National University \\ College of Medicine, Seoul, Korea
}

Background and Purpose Thrombectomy within 24 hours can improve outcomes in selected patients with a clinical-infarct mismatch. We devised an easy-to-use visual estimation tool that allows infarct volume estimation in centers with limited resources.

Methods We identified 1,031 patients with cardioembolic or large-artery atherosclerosis infarction on diffusion-weighted images (DWIs) obtained before recanalization therapy and within 24 hours of onset, and occlusion of the internal carotid or middle cerebral artery. Acute DWIs were mapped onto a standard template and used to create visual reference maps with known lesion volumes, which were then used in a validation study (with 130 cases) against software estimates of infarct volume.

Results The DWI reference map chart comprises 144 maps corresponding to 12 different infarct volumes $(0.5,1,2,3,5,7,9,11,13,15,17$, and $19 \mathrm{~mL}$ ) in each of 12 template slices (Montreal Neurological Institute z-axis -15 to $51 \mathrm{~mm}$ ). Infarct volume in a patient is estimated by selecting a slice with a similar infarct size at the corresponding z-axis level on the reference maps and then adding up over all slices. The method yielded good correlations to software volumetrics and was easily learned by both experienced and junior physicians, with approximately 1 to 2 minutes spent per case. The sensitivity, specificity, and accuracy for detecting threshold infarct volumes $(<21,<31$, and $<51 \mathrm{~mL}$ ) were very high (all about $>90 \%$ ).
Correspondence: Dong-Eog Kim Department of Neurology, Dongguk University llsan Hospital, Dongguk University College of Medicine, 27 Dongguk-ro, llsandong-gu, Goyang 10326, Korea

Tel: +82-31-961-7207

Fax: +82-31-961-7213

E-mail:kdongeog@duih.org

*These authors contributed equally to the manuscript as first author.

Received: November 12, 2018 Revised: December 28, 2018 Accepted: January 8, 2019 
Conclusions We developed easy-to-use reference maps that allow prompt and reliable visual estimation of infarct volumes for triaging patients to thrombectomy in acute stroke.

Keywords Diffusion magnetic resonance imaging; Cerebral infarction; Thrombectomy; Infarct volume; Reference standards

\section{Introduction}

The Diffusion-weighted Imaging or Computed Tomography Perfusion Assessment with Clinical Mismatch in the Triage of Wake Up and Late Presenting Strokes Undergoing Neurointervention with Trevor (DAWN) trial demonstrated the efficacy and safety of endovascular thrombectomy performed 6 to 24 hours after the onset of stroke. ${ }^{1,2}$ The trial included patients with occlusion of the intracranial internal carotid artery or proximal middle cerebral aretery, who had a mismatch between the severity of the clinical deficit and the infarct volume. Infarct volume was assessed by processing diffusionweighted magnetic resonance images (diffusion-weighted images [DWIs]) or perfusion computed tomography images with automated software. The cutoff infarct volumes in the definition of mismatch were 21,31 , and $51 \mathrm{~mL}$, depending on the National Institutes of Health Stroke Scale scores ( $\geq 10$ or $\geq 20$ ) and age ( $<80$ or $\geq 80$ years). ${ }^{\text {. }}$

However, software packages that quantify infarct volume may not be affordable to every stroke center worldwide. Appropriately triaging a patient to the correct group for thrombectomy does not require an absolute infarct volume estimate; it only requires choosing the correct volume category. Thus, we hypothesized that an easy-to-use visual estimation tool that allows for the categorization of infarct volumes to clinically useful ranges could be helpful for triaging patients with acute stroke, depending on the (clinical) practice environment of an individual hospital (or stroke center).

We previously provided an objective visual grading system for white matter hyperintensity (WMH). ${ }^{3}$ To facilitate the use of our data as the reference standard for grading $\mathrm{WMH}$, we plotted the data as frequency-volume (FV) maps that demonstrate the statistical volume and spatial distribution in a single format. The system (Kim statistical WMH scoring system) re-

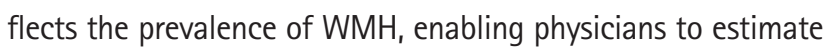
the degree of brain WMH volume as the percentile rank in the stroke population.-5

In the present study, we aimed to develop an easy-to-use reference map system that allows a prompt visual estimation of DWI lesion volumes, with practically acceptable levels of accuracy and precision, for the clinical management of acute stroke patients who can be candidates for mechanical thrombectomy at 6 to 24 hours after onset, based on the results of the DAWN trial.

\section{Methods}

\section{Study population}

This is a multicenter study that involved 11 academic stroke centers participating in the Korean Nationwide Image-based Stroke Database Project. ${ }^{6}$ From May 2011 to February 2014, we consecutively enrolled 1,031 acute stroke patients (Supplementary Figure 1) with (1) cardioembolic or large-artery atherosclerosis infarction on DWIs that were obtained before recanalization therapy and within 24 hours after symptom onset, and (2) occlusion of the internal carotid artery or middle cerebral artery. All patients or their legally authorized representatives provided written informed consent. The Institutional Review Board of Dongguk University Ilsan Hospital approved the project.

\section{Brain magnetic resonance imaging and quantitative image registration}

Brain magnetic resonance imaging (MRI) was performed on a 1.5-T $(n=745)$ or 3.0-T ( $n=286)$ MRI system. The diffusionweighted MRI protocols were as follows: b-values 0 and 1,000 $\mathrm{s} / \mathrm{mm}^{2}$, repetition time 2,400 to $9,000 \mathrm{~ms}$, echo time 50 to 99 $\mathrm{ms}$, voxel size $1 \times 1 \times 3$ to $1 \times 1 \times 5 \mathrm{~mm}^{3}$, interslice gap 0 to $2 \mathrm{~mm}$, and thickness 3 to $7 \mathrm{~mm}$. All images were transferred to the Korean Brain MRI Data Center for central data storage and quantitative analysis. As previously reported, ${ }^{3,4,6}$ DWls were converted to a patient-independent quantitative visual format. Briefly, brain template images $\left(1 \times 1 \times 1 \mathrm{~mm}^{3}\right.$ voxels $)$ were chosen from the Montreal Neurological Institute (MNI) template within the range of -63.5 to $74.5 \mathrm{~mm}$ in the z-axis of the Talairach space. After the normalization of images, each patient's high-signal-intensity lesions on DWIs were semiautomatically segmented and registered onto the brain templates, under meticulous supervision by a vascular neurologist (W.S.R.). The infarct percentage (per brain parenchymal volume) on DWls was calculated as a percentage of brain volume by dividing the number of voxels in the lesions over the total number of parenchymal brain voxels, with corrections applied to account for 
the differences in scan slice thicknesses by adjusting the denominators. ${ }^{3,46}$ Considering that the reported mean brain volume of an elderly Korean population was 1,170 $\mathrm{mL}_{1}^{7}$ the total infarct volume in each patient's registration data was approximated as follows: infarct percentage $\times 1,170 / 100$.

\section{Statistical anatomic maps: DWI lesion FV maps}

To display our data as visual maps that could be used as a reference for estimating DWI lesion volume, we used $12 \mathrm{MNI}$ template slices (centered on $-15,-9,-3,3,9,15,21,27,33$, 39,45 , and $51 \mathrm{~mm}$ on the z-axis) covering the levels of the striatocapsular, corona radiata, and centrum semiovale regions. These slices were chosen, on the basis of clinical experience, as being useful in the estimation of anterior circulation infarct volume, and are representative visual images of a larger data set comprising the whole brain. ${ }^{3}$ First, we plotted the frequency (incidence) of DWI lesions at every voxel coordinate of each slice as a heat map. Then, we plotted the data as FV maps to show the statistical volume and spatial distribution in a single format, arranged to reflect the distribution of disease in our study population, as previously reported, ${ }_{1}^{3}$ with some modifications. The 256 color-coded FV maps were processed to generate the final red color-overlaid "reference maps."

\section{Visual estimation of infarct volume with the reference maps}

By using the reference maps that correspond to 12 different infarct volumes $(0.5,1,2,3,5,7,9,11,13,15,17$, and $19 \mathrm{~mL})$ in each of the 12 template slices, an experienced vascular neurologist (W.S.R.) and a first-year neurology resident (P.K.) independently estimated infarct volumes in 130 patients who were randomly chosen from the Korean Nationwide Image-based Stroke Database Project. For DWls with 5 to $7 \mathrm{~mm}$ thickness (total number of slices approximately 24), all 12 supratentorial slices were used. For DWls with $3 \mathrm{~mm}$ thickness, half of all supratentorial slices $(n=12)$ were used, based on the proximity to the template slices. In the selection of the closest reference image, the first step was to find the DWI slice with the first appearance of the lateral ventricle, corresponding to slice \#5 in the reference maps. Once the first matching was done, the other matchings were subsequently determined by the DWI slice thickness itself; that is, every consecutive reference image for 5 to $7 \mathrm{~mm}$ thickness versus every other consecutive reference image for 2 to $4 \mathrm{~mm}$ thickness. For each DWI slice, infarct volume was estimated by selecting a slice with a similar infarct size at the corresponding z-axis level in the reference maps. If the total infarct volume in a slice was $<0.5 \mathrm{~mL}$, the estimated infarct volume in the slice was assigned as $0.3 \mathrm{~mL}$. All infarct slice volumes were then summed to obtain the "estimated infarct volume" in each patient. To generate a learning curve for the estimation of infarct volume based on the reference maps, we measured the time spent on estimating the infarct volume.

\section{Calculation of infarct volumes with the original DWls}

A vascular neurologist (H.G.J.) who was blinded to the study hypothesis computed the "measured infarct volume" in each patient by using Analyze 12.0, a software package for biomedical image analysis (Biomedical Imaging Resource, New York, NY, USA). The regions of interest were segmented using the Region Grow in the Volume Edit module, with manual removal of artifacts when necessary. The total volume was reported in $\mathrm{mL}$.

\section{Statistical analysis}

We compared the "estimated infarct volume" and the "measured infarct volume" by using Pearson correlation analysis and Bland-Altman plots. We also calculated the sensitivity, specificity, and accuracy of the reference map-based estimation of infarct volumes in terms of the classification of the infarct volumes as $<21,<31$, and $<51 \mathrm{~mL}$. In addition, we included the cutoff point of $70 \mathrm{~mL}$ to also reflect the eligibility criteria of the Endovascular Therapy following Imaging Evaluation for Ischemic Stroke (DEFUSE 3) trial. ${ }^{8}$ A $P<0.05$ was considered statistically significant. All analyses were performed using STATA version 14.0 (STATA Corp., College Station, TX, USA).

\section{Results}

\section{Patients}

In this study on 1,031 patients with acute ( $<24$ hours) cerebral infarction due to the occlusion of the anterior circulation large arteries, the mean age was $70.4 \pm 12.4$ years and $55 \%$ of the patients were men (Table 1). The occluded vessel was the middle cerebral artery in 573 patients (56\%) and the internal carotid artery in 458 patients (44\%). The stroke subtype was large-artery atherosclerosis in 579 patients (56\%) and cardioembolism in 452 patients (44\%). The median onset-to-imaging time was 14 hours (interquartile range, 6 to 21). The median infarct volume was $0.93 \%$ (interquartile range, $0.29 \%$ to $4.18 \%$ ) of the total brain volume, which corresponds to 10.9 $\mathrm{mL}$ (interquartile range, 3.4 to 48.9 ).

\section{Reference maps}

Supplementary Figure 2 shows the results of a combined mapping of both spatial extent and frequency of occurrence of 
Table 1. Baseline characteristics of 1,031 patients with acute stroke due to anterior circulation large-artery occlusion

\begin{tabular}{lc}
\hline Characteristic & Acute $(<24 \mathrm{hr})$ cerebral infarction $(\mathrm{n}=1,031)$ \\
\hline Age $(\mathrm{yr})$ & $70.4 \pm 12.4$ \\
Male sex & $563(54.6)$ \\
Hypertension & $730(70.8)$ \\
Diabetes & $345(33.5)$ \\
Hyperlipidemia & $357(34.6)$ \\
Smoking, current or quit $<5 \mathrm{yr}$ & $419(40.6)$ \\
Atrial fibrillation & $406(39.4)$ \\
Coronary artery disease & $164(15.9)$ \\
Stroke subtype & \\
Large-artery atherosclerosis & $579(56.2)$ \\
Cardioembolism & $452(43.8)$ \\
Occluded artery & \\
Middle cerebral artery & $573(55.6)$ \\
Internal carotid artery & $458(44.4)$ \\
NIHSS & $99(4-15)$ \\
Time from ictus to imaging (hr) & $14(6-21)$ \\
Infarct volume (percent of brain volume) & $0.93(0.29-4.18)$ \\
\hline
\end{tabular}

Values are presented as mean \pm SD, number $(\%)$, or median (interquartile range).

NIHSS, National Institutes of Health Stroke Scale.

acute DWI lesions, and Figure 1 shows the final reference maps. The number above each brain image indicates the corresponding infarct volume in the "hemisphere" (or one-half) of the brain. As aforementioned, the estimation of infarct volume on a clinical DWI section could be performed by selecting a slice with a similar infarct size at the corresponding z-axis level in the reference maps. If a DWI section has multiple infarcts with different volumes, the total lesion volume in the section may have to be calculated by finding multiple slices that represent the different volumes at the same z-axis level.

The color coding within each infarcted volume in Supplementary Figure 2 represents the hemisphere-wise infarct frequency at each voxel, reflecting that a red (vs. blue) voxel had a higher (vs. lower) number of patients with a lesion in the voxel.

\section{Visual estimation of infarct volume with the reference maps vs. computer-assisted volume measurement with an image analyzer}

The "estimated infarct volumes" that were obtained by a vascular neurologist using reference maps correlated well to the "measured infarct volumes" that were obtained by another vascular neurologist using the Analyze software $(r=0.977$, $P<0.001$ ) (Figure 2A). A Bland-Altman plot showed good agreement between the two methods; only six of the 130 cases
(4.6\%) were outside the limits of agreement (Figure 2B). The disagreement was more prominent when infarct volumes were larger; if the infarct volumes measured using the Analyzebased method were $>50 \mathrm{~mL}$, the reference map-based method estimated them to be about $40 \%$ smaller.

When we divided the patients according to the DWI slice thickness $(3,5,6$, and $7 \mathrm{~mm})$, a Bland-Altman plot again showed good agreement between the two methods, although the reference map-based method again tended to underestimate infarct volumes when they were large (Supplementary Figure 3).

The sensitivity, specificity, and accuracy of the reference map-based estimation of infarct volumes in terms of the classification of the infarct volumes as $<21,<31$, and $<51 \mathrm{~mL}$ were high $(88.9 \%, 100 \%$, and $95.4 \% ; 85.1 \%, 100 \%$, and $94.6 \%$; and $86.5 \%, 100 \%$, and 96.2\%, respectively) (Table 2 and Figure 2A). The kappa value between the classification by the two methods was $0.75(P<0.001)$, indicating a good agreement. In addition, the sensitivity, specificity, and accuracy of the reference map-based estimation of infarct volumes in terms of the classification of the infarct volumes as $<70 \mathrm{~mL}$ versus $\geq 70 \mathrm{~mL}$ were also high $(77.8 \%, 100 \%$, and $95.4 \%$, respectively).

As described before, we observed that infarct volumes estimated using the reference maps tended to be smaller than infarct volumes measured using an image analyzer. To improve the performance of the infarct volume estimation and classifi- 
Slice \#1, $6 \mathrm{~mm}$ thickness
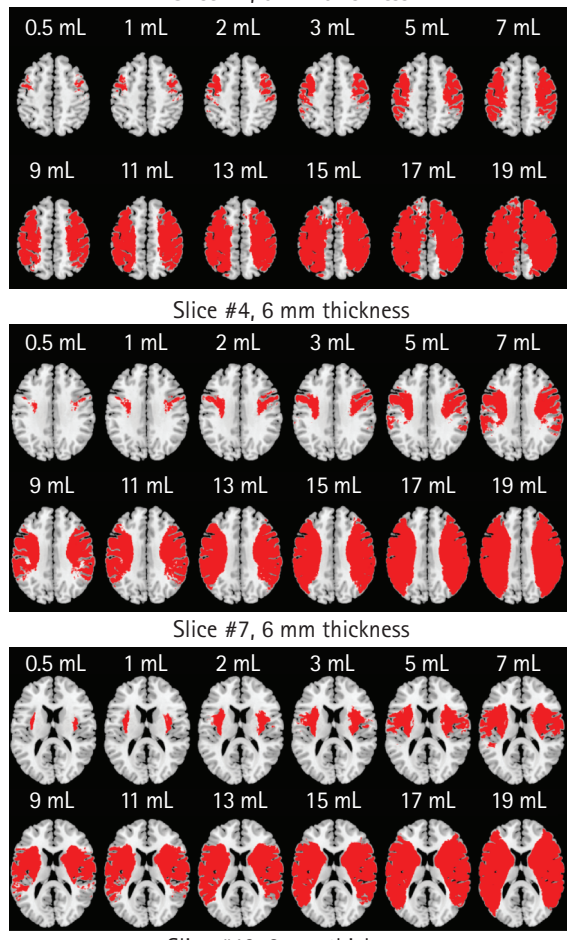

Slice \#10, $6 \mathrm{~mm}$ thickness
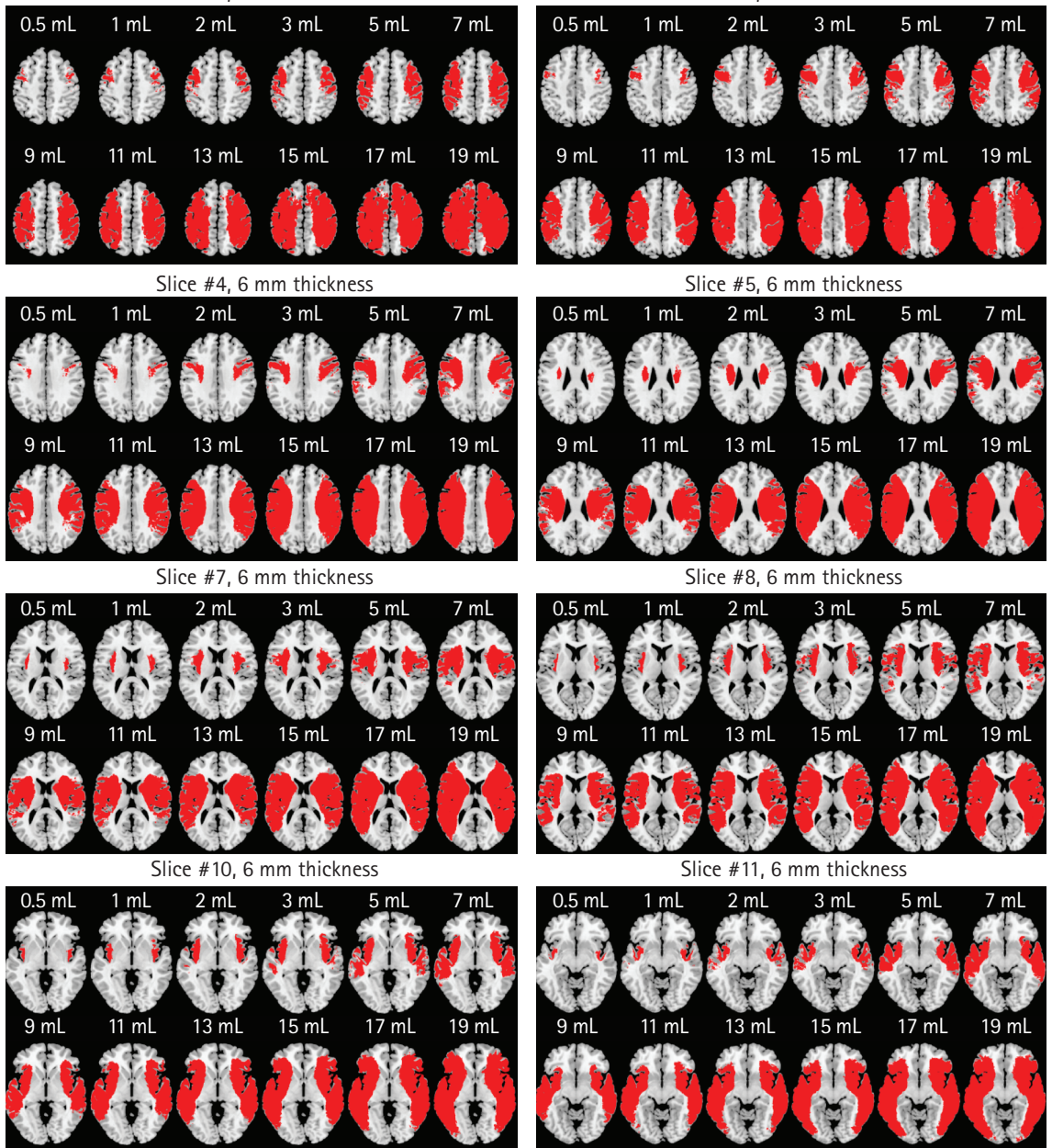

Slice \#11, $6 \mathrm{~mm}$ thickness

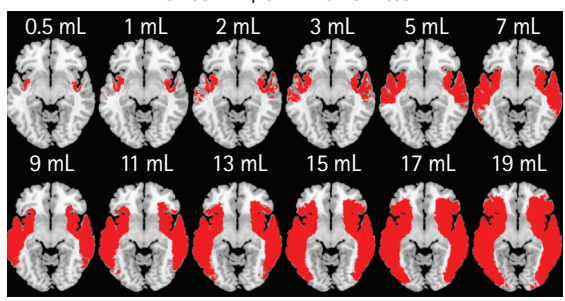

As a post hoc adjustment to improve the accuracy,

multiply the sum of all estimated infarct volumes by $1.1,1.2,1.3$, or 1.4 for the sum value of $<21,21$ to $<31,31$ to $<51$, or $51 \mathrm{~mL}$, respectively.
Slice \#3, $6 \mathrm{~mm}$ thickness

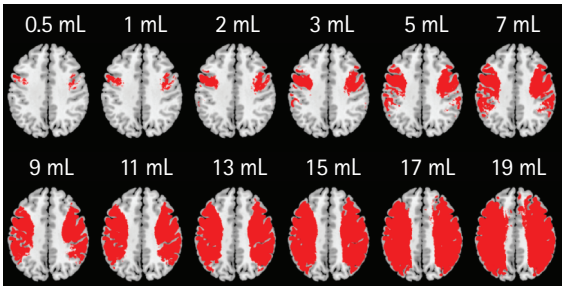

Slice \#6, $6 \mathrm{~mm}$ thickness

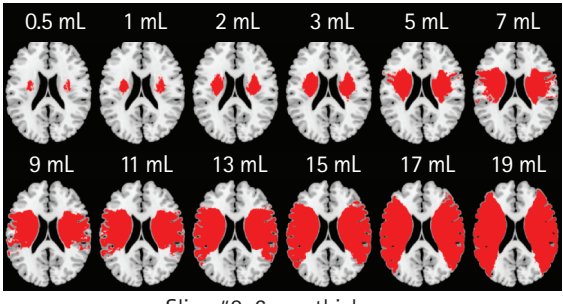

Slice \#9, $6 \mathrm{~mm}$ thickness

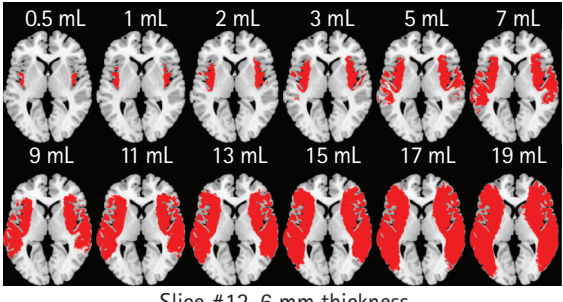

Slice \#12,6 mm thickness

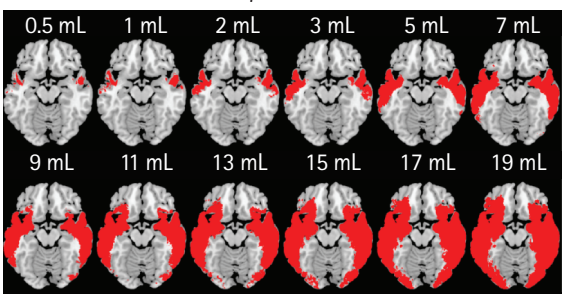

Figure 1. Reference maps for visual estimation of acute infarct volume. The topographic frequency-volume maps of acute diffusion-weighted imaging (DWI) lesions (Supplementary Figure 2) were processed to generate these final reference maps. The number above each brain image indicates the corresponding infarct volume $(\mathrm{mL})$ in one "hemisphere" of the brain; the lesion volumes of both hemispheres on each brain image are the same. The thickness of the map slices is $6 \mathrm{~mm}$. The visual estimation of infarct volume on a clinical DWI section is performed by selecting a slice with a similar infarct size at the corresponding zaxis level in the reference maps. After all infarct volumes in every slice are summed, the formula at the bottom of this figure is used for adjusting the sum value to improve the accuracy.

cation based on the reference maps, we generated the following simple adjustment formula after considering the aforementioned infarct volume-related non-linear pattern of the errors as well as the cutoff DWI infarct volumes $(21,31$, and $51 \mathrm{~mL})$ : multiply the sum of all estimated infarct volumes by 1.1, 1.2, 1.3 , or 1.4 for the sum value of $<21,21$ to $<31,31$ to $<51$, or $\geq 51 \mathrm{~mL}$, respectively. The adjusted estimated infarct volumes better approximated to the measured infarct volumes (Figure $3 A)$. The mean difference in the infarct volumes that were provided by the reference map-based method versus the Analyzebased method decreased from 14.5 to $2.6 \mathrm{~mL}$. Only five cases (3.8\%) were outside the limits of agreement (Figure 3B). Accordingly, the sensitivity, specificity, and accuracy of the refer- ence map-based estimation of infarct volumes in terms of the classification of the infarct volumes as $<21,<31$, and $<51 \mathrm{~mL}$ became higher $(90.7 \%, 100 \%$, and $96.2 \% ; 93.6 \%, 98.8 \%$, and 96.9\%; and 100\%, 100\%, and 100\%, respectively) (Figure $3 \mathrm{~A}$ and Supplementary Table 1). The kappa value between the classification by the two methods was $0.89(P<0.001)$, indicating an excellent agreement. In addition, the sensitivity, specificity, and accuracy of the reference map-based estimation of infarct volumes in terms of the classification of the infarct volumes as $<70 \mathrm{~mL}$ versus $\geq 70 \mathrm{~mL}$ again became higher (92.3\%, $94.2 \%$, and $93.9 \%$, respectively).

The post hoc adjustment-related improvement of the infarct volume estimation was proved in a validation study using a dif- 

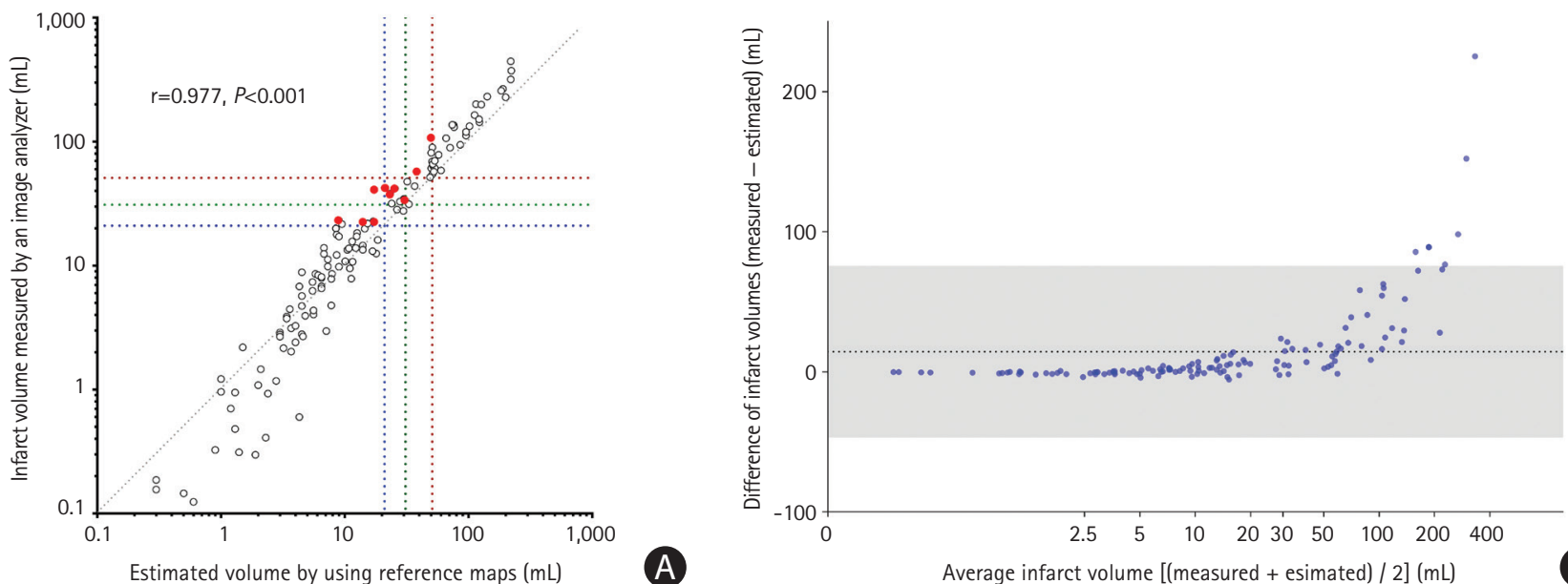

Figure 2. Correlations between estimated vs. measured infarct volumes. (A) Each dot indicates one patient's datum ( $n=130)$, representing a single reader estimate of volume $(\mathrm{mL})$ versus the actual volume $(\mathrm{mL})$. The clustering close to the cutoffs suggests a "categorical" effect. Blue, green, and red dotted lines indicate infarct volume thresholds of 21,31, and $51 \mathrm{~mL}$. The red dots indicate the data that were misclassified (for the classification of volumes of $>21$, $>31$, and $>51 \mathrm{~mL}$ ) by the visual estimation based on the reference maps (Figure 1) versus the measurement with an image analyzer software package (Analyze 12.0). The gray dotted line indicates a perfect estimation slope $(x=y)$. Owing to a skewed distribution of infarct volumes, the $x$-axis and $y$-axis were logarithmically transformed for better visualization. (B) A Bland-Altman plot showing that the error in estimation increases with infarct volume, and that estimates for larger volumes are biased to underestimation. The dotted line indicates the mean difference between the estimated infarct volumes and the measured infarct volumes. A gray area indicates $95 \%$ limits of agreement. A logarithmic scale is used for the $\mathrm{x}$-axis of the Bland-Altman plot.

Table 2. Categorization of infarct volumes after visual estimation with reference maps vs. measurement with an image analyzer

\begin{tabular}{|c|c|c|c|c|c|}
\hline \multirow{2}{*}{ Reference maps } & \multicolumn{4}{|c|}{ Image analyzer } & \multirow{2}{*}{ Total } \\
\hline & $<21 \mathrm{~mL}$ & 21 to $<31 \mathrm{~mL}$ & 31 to $<51 \mathrm{~mL}$ & $\geq 51 \mathrm{~mL}$ & \\
\hline$<21 \mathrm{~mL}$ & 76 & 5 & 1 & 0 & 82 \\
\hline 21 to $<31 \mathrm{~mL}$ & 0 & 2 & 6 & 0 & 8 \\
\hline 31 to $<51 \mathrm{~mL}$ & 0 & 0 & 3 & 6 & 9 \\
\hline$\geq 51 \mathrm{~mL}$ & 0 & 0 & 0 & 31 & 31 \\
\hline Total & 76 & 7 & 10 & 37 & 130 \\
\hline
\end{tabular}

ferent set of data $(n=30)$. The adjusted estimated infarct volumes again closely correlated with the measured infarct volumes $(r=0.979, P<0.001)$ (Supplementary Figure 4). A Bland-Altman plot again showed a high level of agreement between the two methods; only three of the 30 cases (10\%) were outside the limits of agreement (Supplementary Figure 5). The sensitivity, specificity, and accuracy of the reference map-based estimation of infarct volumes in terms of the classification of the infarct volumes as $<21,<31$, and $<51 \mathrm{~mL}$ were also high $(93.8 \%$, 92.9\%, and 93.3\%; 91.7\%, 94.4\%, and 93.3\%; and 100\%, 100\%, and 100\%, respectively) (Supplementary Table 2 and Supplementary Figure 4). The kappa value between the classification by the two methods was 0.80 ( $P<0.001)$. In addition, the sensitivity, specificity, and accuracy of the reference map-based estimation of infarct volumes in terms of the classification of the infarct volumes as $<70 \mathrm{~mL}$ versus $\geq 70 \mathrm{~mL}$ were all $100 \%$.

\section{Visual estimation of infarct volumes by a vascular} neurologist vs. that by a first-year resident

The infarct volumes estimated by a vascular neurologist and a first-year resident using the reference maps were reliable when assessed using a Bland-Altman plot; 10 of 130 (7.7\%) patients were outside the limits of agreement, and the mean difference between the two raters was $0.8 \mathrm{~mL}$ (Supplementary Figure 6). In addition, the correlation plot between the estimated volumes by the first-year resident and the measured volumes appeared similar to that by the vascular neurologist (Supplementary Figure 7). The times taken for estimating infarct volumes were short (1 to $2 \mathrm{~min} /$ case) and showed a decreasing trend over experiences: $42,41,36,30,31$, and 29 minuts per 20 patients for the vascular neurologist versus 29, 25, 26, 24, 20, and 16 minutes per 20 patients for the first-year resident (Supplementary Figure 8). 

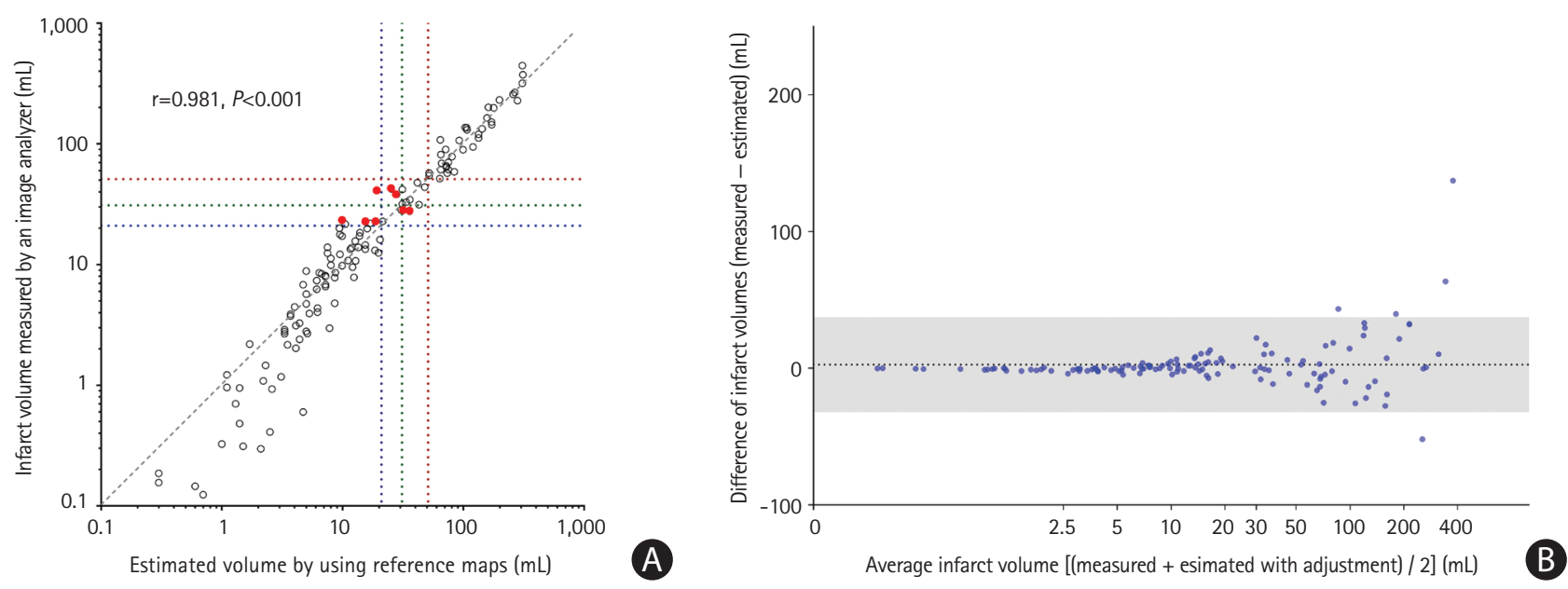

Figure 3. Closer correlations between "adjusted" estimated infarct volumes versus measured infarct volumes. (A) Each dot indicates one patient's datum. Blue, green, and red dotted lines indicate infarct volume thresholds of 21,31, and $51 \mathrm{~mL}$. The red dots indicate the data that were misclassified (for the classification of volumes of $>21,>31$, and $>51 \mathrm{~mL}$ ) by the visual estimation based on the reference maps and the post hoc adjustment (Figure 1) versus the measurement with an image analyzer software package (Analyze 12.0). The gray dotted line indicates a perfect estimation slope ( $\mathrm{x}=\mathrm{y})$. Owing to a skewed distribution of infarct volumes, the $x$-axis and $y$-axis were logarithmically transformed for better visualization. (B) A Bland-Altman plot. The dotted line indicates the mean difference between the adjusted estimated infarct volumes and the measured infarct volumes. A gray area indicates $95 \%$ limits of agreement. A logarithmic scale is used for the $\mathrm{x}$-axis of the Bland-Altman plot.

\section{Discussion}

In the present study, we developed reference maps and showed the potential clinical utility of the map chart that enables the visual estimation of infarct volumes (Supplementary Video 1) in patients with acute supratentorial ischemic stroke due to large-artery atherosclerotic or cardioembolic occlusion of the middle cerebral artery or internal carotid artery. There was a close correlation between the reference map-based visual estimation of infarct size and the image analyzer-based volume measurement, particularly after applying the post hoc adjustment formula to our map-based method. The estimated infarct volumes correlated well between a vascular neurologist and a first-year resident, and it took only 1 to 2 minutes for the estimation of one patient's DWls after a short learning period. In addition, the threshold infarct volumes used in previous randomized clinical trials for image-based thrombectomy were well differentiated by using the reference maps.

This study has limitations. First, we did not compare the estimation of infarct volume by using the reference maps with the volume measurement by using the identical software packages used in clinical trials for image-based thrombectomy. ${ }^{1}$ However, the software used (Analyze) has a long history in multiple publications, ${ }^{9-11}$ and would be expected to perform similarly to that used in the DAWN trial.

Second, our findings are limited to DWI infarct estimation; further investigations are required to study whether the reference maps could be used for the measurement of perfusion CT- derived infarct core volumes.

Third, the reference map-based method tended to underestimate infarct volumes. The reference maps were generated using the registered images on the MNI template set after normalization of the original DWIs and warping of segmented infarct lesions. The use of 1,170 $\mathrm{mL}$ as the brain volume as well as the image registration-related process may be partly responsible for the disagreement; we speculate that the average brain volume in our study population may be $>1,170 \mathrm{~mL}$. Hence, we devised an adjustment formula, which improved the performance of the visual estimation of infarct volume with our map-based method. The post hoc adjustment seems rather arbitrary; however, the purpose of this study was to develop an easy-to-use reference map system that allows a prompt estimation of DWI lesion volumes with practically acceptable levels of accuracy and precision. Thus, it is notable that after the adjustment, the sensitivity, specificity, and accuracy of the map-based visual estimation of infarct volumes in terms of the classification of the infarct volumes as $<21,<31$, and $<51 \mathrm{~mL}$ (i.e., the cutoff infarct volumes; see also Figure 4: maps that are representative of 21,31 , and $51 \mathrm{~mL}$ infarct volumes) were almost $90 \%$ to $100 \%$. In addition, the DEFUSE criteria (70 mL cutoff) could also be easily accommodated. The post hoc adjustment still cannot overcome the inherent limitation that is related to our approach based on the estimation of an absolute lesion volume by multiplying a lesion-to-brain ratio with a fixed number: the average brain volume. However, choosing the correct volume category, which is currently required for tri- 


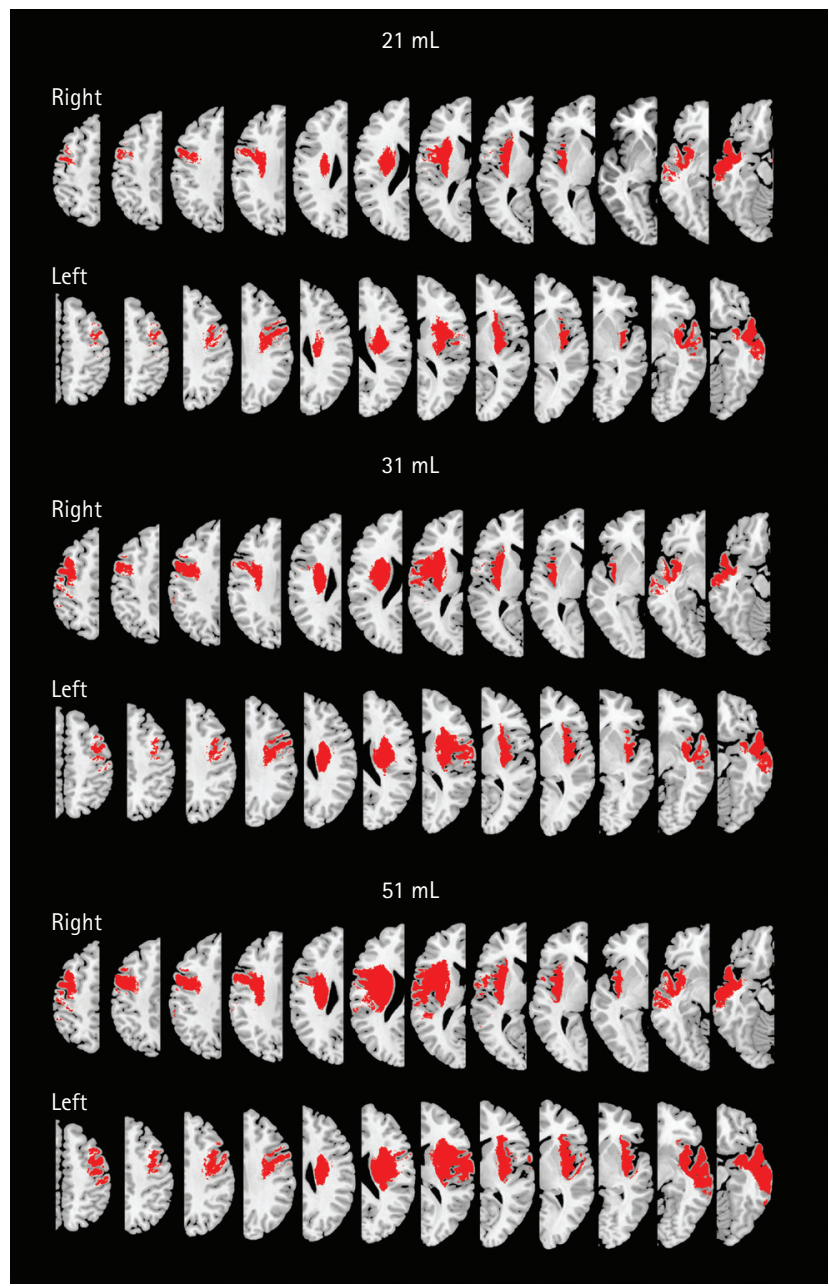

Figure 4. Reference maps that are representative of the infarct volumes of 21,31 , and $51 \mathrm{~mL}$. Representative reference maps that correspond to 21 $\mathrm{mL}$ (upper), $31 \mathrm{~mL}$ (middle), and $51 \mathrm{~mL}$ (lower) infarct volumes (adjusted values; see the main text for the adjustment formula). The maps reflect not only volumetric information but also the spatial distribution that represents the voxel-wise lesion frequency in the stroke population of this study.

aging patients to the correct group for thrombectomy, may be less stringent than securing an absolute infarct volume estimate.

Fourth, the results of this study may not be directly applicable to other centers or ethnic groups. The thickness of DWls in our multicenter cohort was mostly 6 or $3 \mathrm{~mm}$. Thus, the clinically acceptable validity of our method might possibly be impeded when a very different slice thickness is used as a scanning protocol of DWls.

Despite the limitations, we believe that the reference mapbased estimation of DWI lesion volumes has practically acceptable levels of accuracy and precision, allowing for thrombectomy-related clinical management of patients with acute stroke in centers with limited resources.

\section{Supplementary materials}

Supplementary materials related to this article can be found online at https://doi.org/10.5853/jos.2018.03202.

\section{Disclosure}

The authors have no financial conflicts of interest.

\section{Acknowledgments}

This study was supported by grants from the National Center for Standard Reference Data, Korean Agency for Technology and Standards, Ministry of Trade, Industry \& Energy, Ministry of Health \& Welfare (HI12C1847; Korea Healthcare Technology R\&D Project), and the Global Research Lab program (NRF2015K1A1A2028228) of the National Research Foundation, funded by the Korean government, Republic of Korea.

We appreciate the contributions of all members of the Clinical Research Center for Stroke in Korea (CRCS-K) to this study.

\section{References}

1. Nogueira RG, Jadhav AP, Haussen DC, Bonafe $A$, Budzik RF, Bhuva $P$, et al. Thrombectomy 6 to 24 hours after stroke with a mismatch between deficit and infarct. N Engl J Med 2018; 378:11-21.

2. Lee KJ, Kim BJ, Kim DE, Ryu WS, Han MK, Kim JT, et al. Nationwide estimation of eligibility for endovascular thrombectomy based on the DAWN trial. JStroke 2018;20:277-279.

3. Ryu WS, Woo SH, Schellingerhout D, Chung MK, Kim CK, Jang $\mathrm{MU}$, et al. Grading and interpretation of white matter hyperintensities using statistical maps. Stroke 2014;45:3567-3575.

4. Ryu WS, Woo SH, Schellingerhout D, Jang MU, Park KJ, Hong $\mathrm{KS}$, et al. Stroke outcomes are worse with larger leukoaraiosis volumes. Brain 2017;140:158-170.

5. Kim DE, Park JH, Schellingerhout $D$, Ryu WS, Lee SK, Jang $\mathrm{MU}$, et al. Mapping the supratentorial cerebral arterial territories using 1160 large artery infarcts. JAMA Neurol 2019; 76:72-80.

6. Kim DE, Park KJ, Schellingerhout $D$, Jeong SW, Ji MG, Choi WJ, et al. A new image-based stroke registry containing quantitative magnetic resonance imaging data. Cerebrovasc Dis 2011;32:567-576.

7. Kwon JY, Rhyu IJ, Cheon JJ, Koh IS. Brain volume measurement of Healthy Korean for clinical application using MRI. Rep Natl Inst Health 2001;38:279-284.

8. Albers GW, Marks MP, Kemp S, Christensen S, Tsai JP, Ortega- 
Gutierrez S, et al. Thrombectomy for stroke at 6 to 16 hours with selection by perfusion imaging. N Engl J Med 2018;378: 708-718.

9. Barrett KM, Ding YH, Wagner DP, Kallmes DF, Johnston KC; ASAP Investigators. Change in diffusion-weighted imaging infarct volume predicts neurologic outcome at 90 days: results of the Acute Stroke Accurate Prediction (ASAP) trial serial imaging substudy. Stroke 2009;40:2422-2427.

10. Boers AM, Marquering $H A$, Jochem JJ, Besselink NJ, Berkhemer
$O A$, van der Lugt $A$, et al. Automated cerebral infarct volume measurement in follow-up noncontrast CT scans of patients with acute ischemic stroke. AJNR Am J Neuroradiol 2013;34: 1522-1527.

11. Zaidi SF, Aghaebrahim A, Urra X, Jumaa MA, Jankowitz B, Hammer $M$, et al. Final infarct volume is a stronger predictor of outcome than recanalization in patients with proximal middle cerebral artery occlusion treated with endovascular therapy. Stroke 2012;43:3238-3244. 
Supplementary Video 1. Illustration of the reference map-based visual estimation of infarct volume. 
Supplementary Table 1. Improved categorization of infarct volumes after adjustment* of the reference map-based volume estimation versus volume measurement by using an image analyzer

\begin{tabular}{lccccc}
\hline \multirow{2}{*}{ Reference maps } & \multicolumn{4}{c}{ Image analyzer } & \multirow{2}{*}{ Total } \\
\cline { 2 - 5 } & $<21 \mathrm{~mL}$ & 21 to $<31 \mathrm{~mL}$ & 31 to $<51 \mathrm{~mL}$ & 0 & 81 \\
\hline$<21 \mathrm{~mL}$ & 76 & 4 & 1 & 0 & 4 \\
21 to $<31 \mathrm{~mL}$ & 0 & 2 & 2 & 0 & 8 \\
$31 \mathrm{to}<51 \mathrm{~mL}$ & 0 & 1 & 0 & 37 & 37 \\
$\geq 51 \mathrm{~mL}$ & 0 & 0 & 10 & 37 & 130 \\
Total & 76 & 7 & 7 & & 4 \\
\hline
\end{tabular}

*Adjustment: multiply the sum of all estimated infarct volumes in each patient by $1.1,1.2,1.3$, or 1.4 for the sum value of $<21,21$ to $<31,31$ to $<51$, or $\geq 51$ $\mathrm{mL}$, respectively. 
Supplementary Table 2. A validation study $(n=30)$ to test the improved categorization of infarct volumes by using the reference map-based volume estimation with adjustment* versus volume measurement by using an image analyzer

\begin{tabular}{lccccc}
\hline \multirow{2}{*}{ Reference maps } & \multicolumn{4}{c}{ Image analyzer } & \multirow{2}{*}{ Total } \\
\cline { 2 - 5 } & $<21 \mathrm{~mL}$ & 21 to $<31 \mathrm{~mL}$ & 31 to $<51 \mathrm{~mL}$ & 0 & 14 \\
\hline$<21 \mathrm{~mL}$ & 13 & 1 & 0 & 0 & 4 \\
21 to $<31 \mathrm{~mL}$ & 1 & 2 & 1 & 4 & 5 \\
31 to $<51 \mathrm{~mL}$ & 0 & 1 & 0 & 7 & 7 \\
$\geq 51 \mathrm{~mL}$ & 0 & 0 & 5 & 7 & 30 \\
Total & 14 & 4 & 4 & 5 \\
\hline
\end{tabular}

*Adjustment: multiply the sum of all estimated infarct volumes in each patient by $1.1,1.2,1.3$, or 1.4 for the sum value of $<21,21$ to $<31,31$ to $<51$, or $\geq 51$ $\mathrm{mL}$, respectively. 
13,875 Patients with ischemic stroke within 7 days of symptom onset were screened from May 2011 to February 2014

8,187 Underwent diffusion MRI $>24$ hours after last normal time or did not have MRI 2,903 Without relevant occlusion of middle cerebral artery of internal carotid artery

2,785 Patients with ischemic stroke who had diffusion-weighted images within 24 hours after the last normal time and occlusion of the anterior circulation large arteries

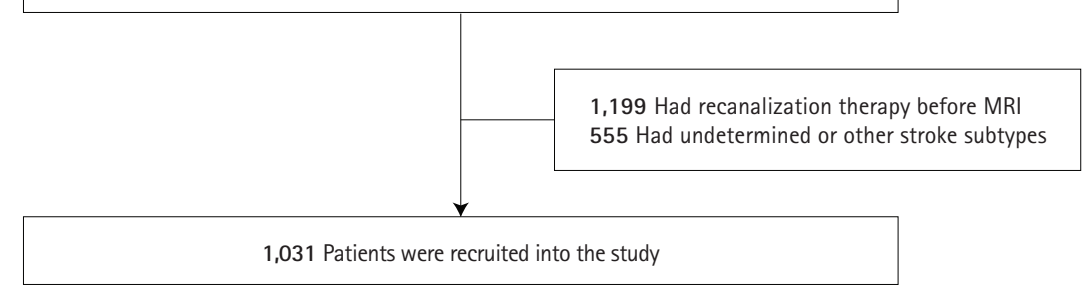

Supplementary Figure 1. Study flowchart. MRI, magnetic resonance imaging. 


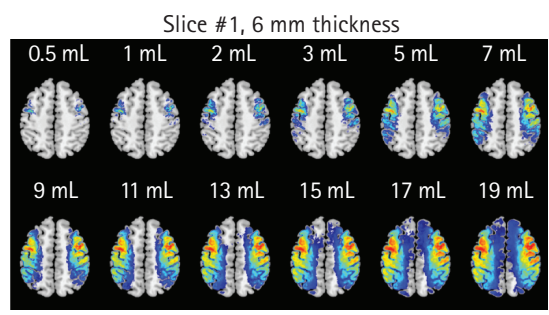

Slice \#4, $6 \mathrm{~mm}$ thickness

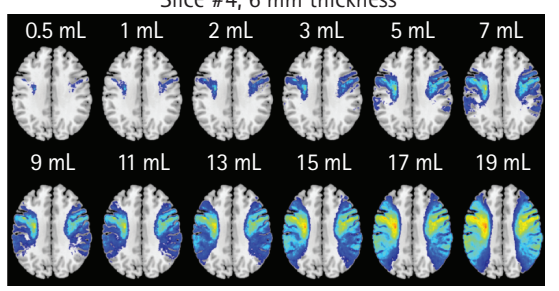

Slice \#7, $6 \mathrm{~mm}$ thickness

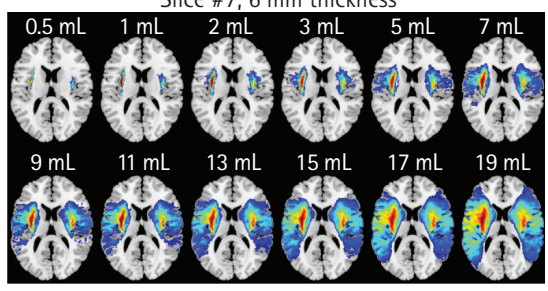

Slice \#10, $6 \mathrm{~mm}$ thickness

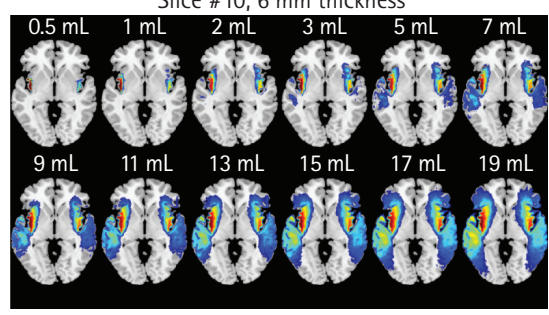

Slice \#2, $6 \mathrm{~mm}$ thickness

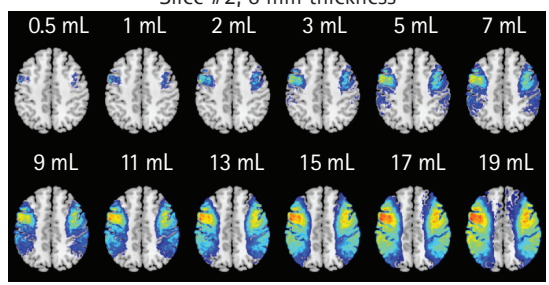

Slice \#5, $6 \mathrm{~mm}$ thickness

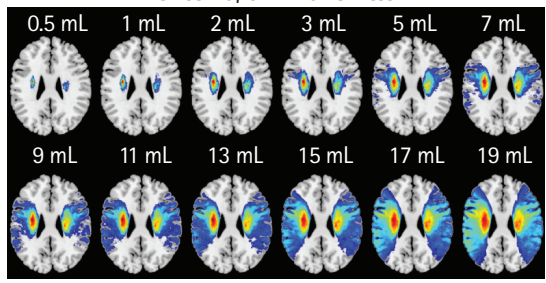

Slice \#8, $6 \mathrm{~mm}$ thickness

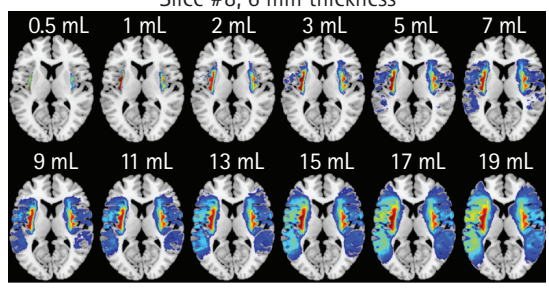

Slice \#11, $6 \mathrm{~mm}$ thickness

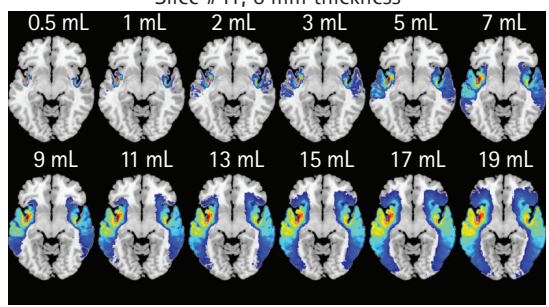

Slice \#3, $6 \mathrm{~mm}$ thickness

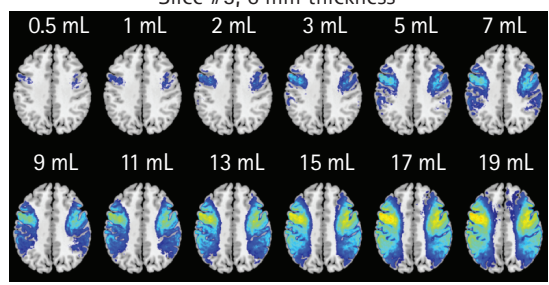

Slice \#6, $6 \mathrm{~mm}$ thickness

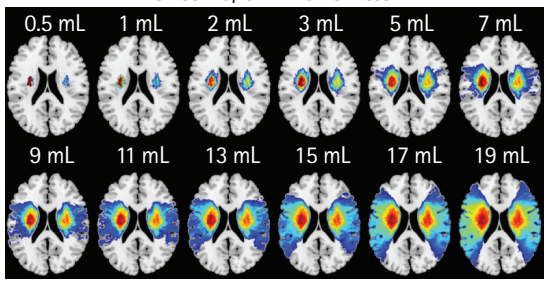

Slice \#9, $6 \mathrm{~mm}$ thickness

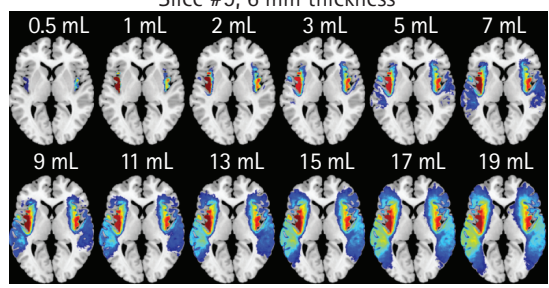

Slice \#12, $6 \mathrm{~mm}$ thickness

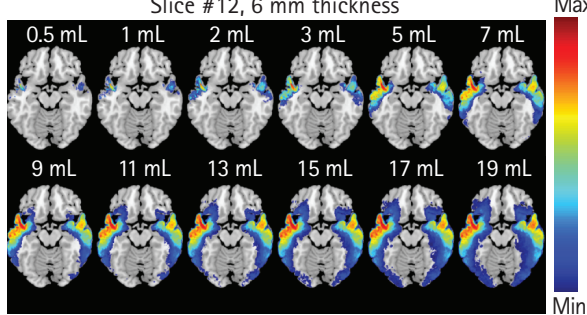

Supplementary Figure 2. Topographic frequency-volume maps to generate the final reference maps (Figure 1) for visual estimation of acute infarct volumes. Combined mapping of both the spatial extent and the frequency of occurrence of acute diffusion-weighted magnetic resonance imaging lesions, with the number above each brain image indicating the corresponding infarct volume $(\mathrm{mL})$ in the "hemisphere" of the brain; the lesion volumes of both hemispheres on each brain image are the same. The color coding represents the hemisphere-wise infarct frequency at each voxel. The thickness of the map slices is $6 \mathrm{~mm}$. Max=maximum frequency. Min=minimum frequency higher than zero. 

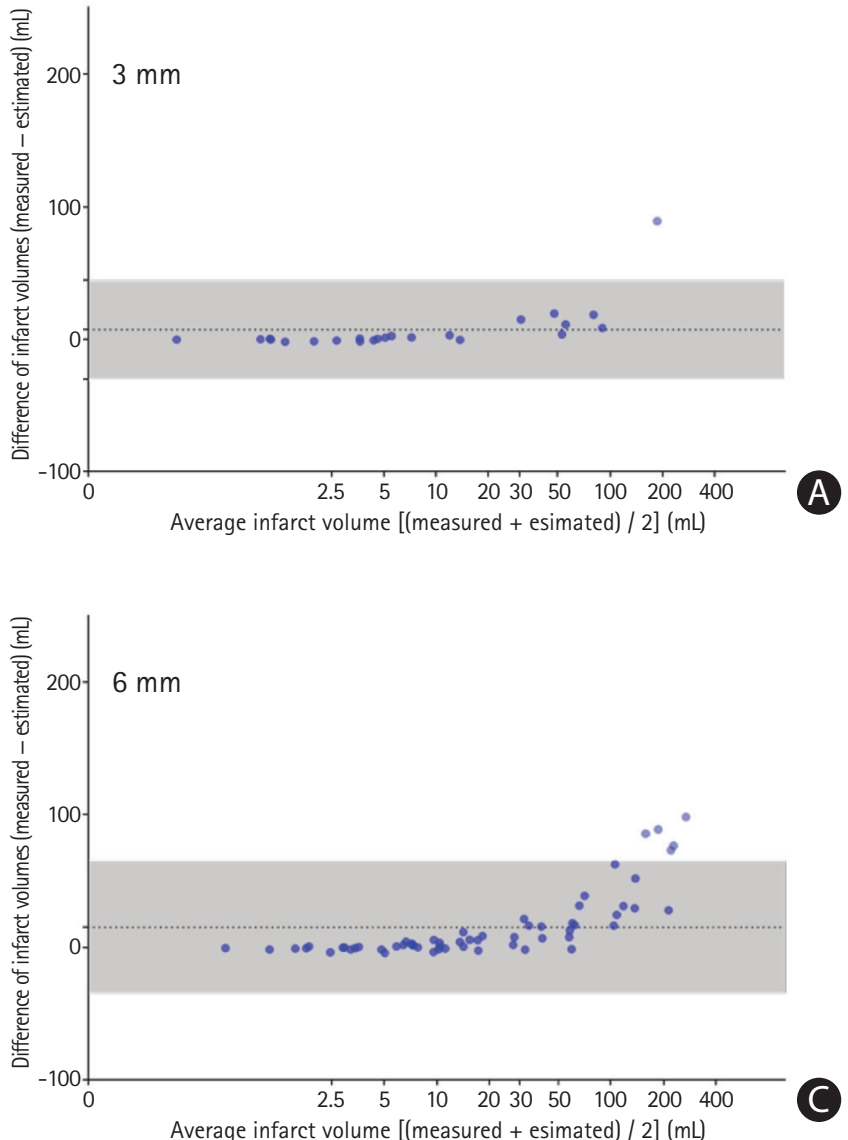

Supplementary Figure 3. Bland-Altman plots, with stratification by the slice thickness of diffusion-weighted magnetic resonance images ( 3 , $3 m$; B, 5 $\mathrm{mm} ; C, 6 \mathrm{~mm} ; \mathrm{D}, 7 \mathrm{~mm}$ ). The dotted line indicates the mean difference between estimated infarct volumes (obtained using the reference maps) and measured infarct volumes (obtained using Analyze 12.0, an image analysis software package). The gray area indicates 95\% limits of agreement. A logarithmic scale is used for the x-axis. The number of patients in each group $(3,5,6$, and $7 \mathrm{~mm})$ was $23,24,59$, and, 24, respectively. The number of patients outside the limits of agreement is $1(4.4 \%), 2(8.3 \%), 5(8.5 \%)$, and $2(8.3 \%)$, respectively.
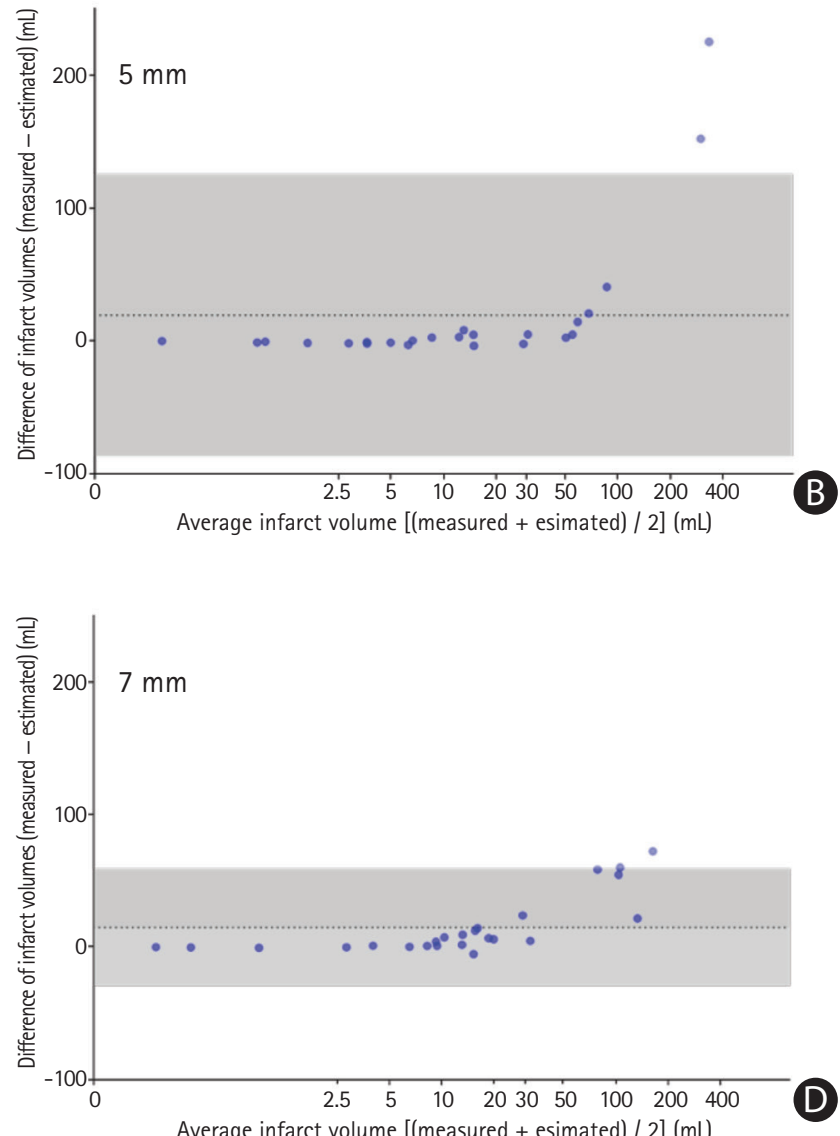

(D) 


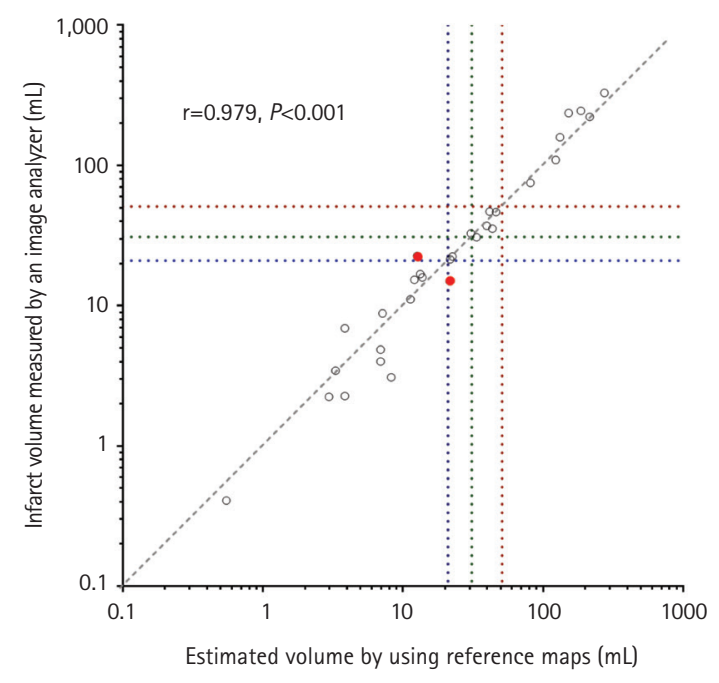

Supplementary Figure 4. Correlations between "adjusted" estimated infarct volumes versus measured infarct volumes. Each dot indicates one patient's datum. Blue, green, and red dotted lines indicate infarct volume thresholds of 21,31 , and $51 \mathrm{~mL}$. The red dots indicate the data that were misclassified (for the classification of volumes of $>21,>31$, and $>51 \mathrm{~mL}$ ) by the visual estimation based on the reference maps and the post hoc adjustment (Figure 1) versus the measurement using an image analyzer (Analyze 12.0). The gray dotted line indicates perfect estimation slope $(x=y)$. Owing to a skewed distribution of infarct volumes, the $x$-axis and $y$-axis were logarithmically transformed for better visualization. 


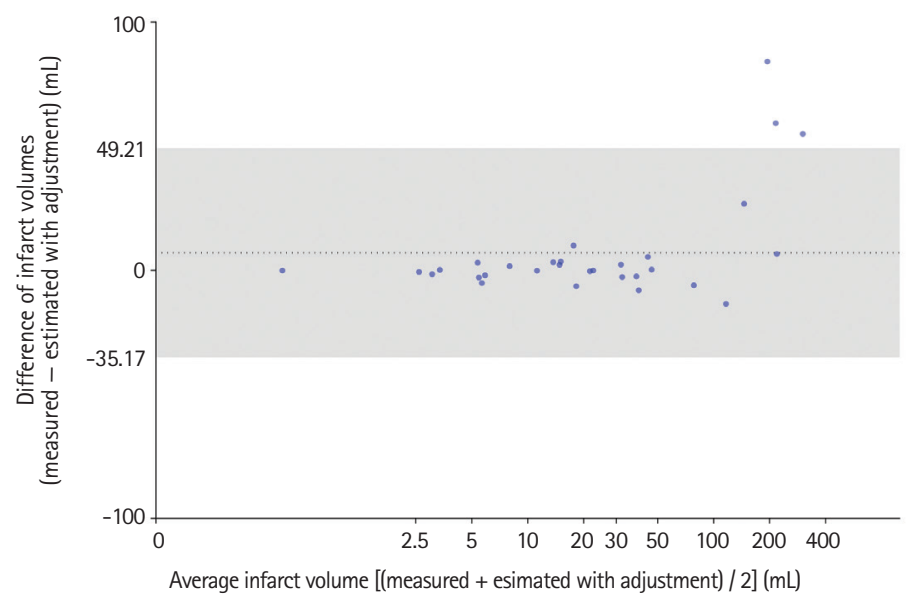

Supplementary Figure 5. A Bland-Altman plot of "adjusted" estimated infarct volumes vs. measured infarct volumes in a validation study ( $\mathrm{n}=30$ ). The dotted line indicates the mean difference between the estimated infarct volumes (obtained using the reference maps with the post hoc adjustment in Figure 1) and the measured infarct volumes (obtained using Analyze 12.0, an image analysis software package). The gray area indicates 95\% limits of agreement. A logarithmicscale is used for the $\mathrm{x}$-axis. Three patients (10\%) are outside the limits of agreement. 


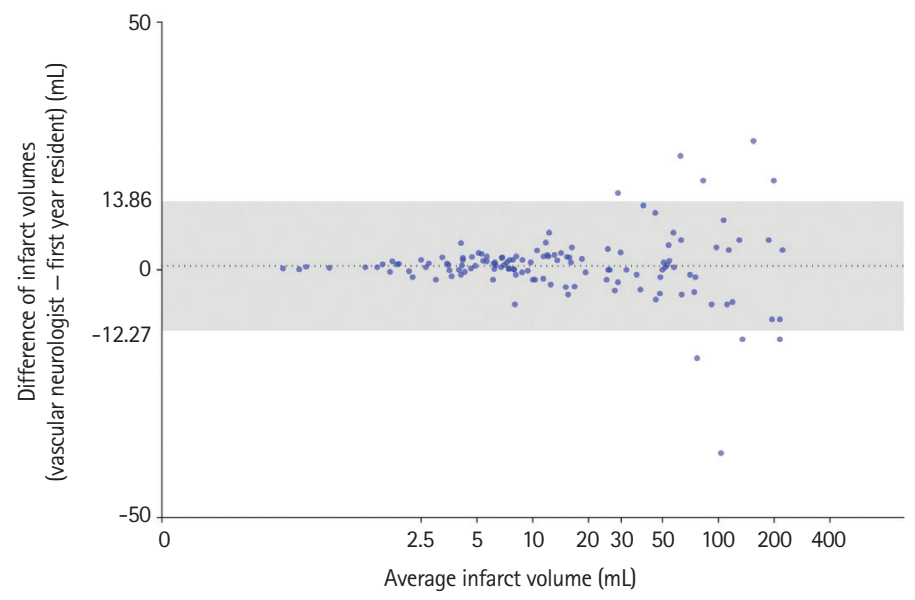

Supplementary Figure 6. A Bland-Altman plot for the reference map-based estimation of infarct volumes by a vascular neurologist versus a first-year neurology resident. The dotted line indicates the mean difference between the estimated infarct volumes by an experienced vascular neurologist and those by a first-year neurology resident. The gray area indicates 95\% limits of agreement. A logarithmic scale is used for the $x$-axis. Ten patients (7.7\%) are outside the limits of agreement. 


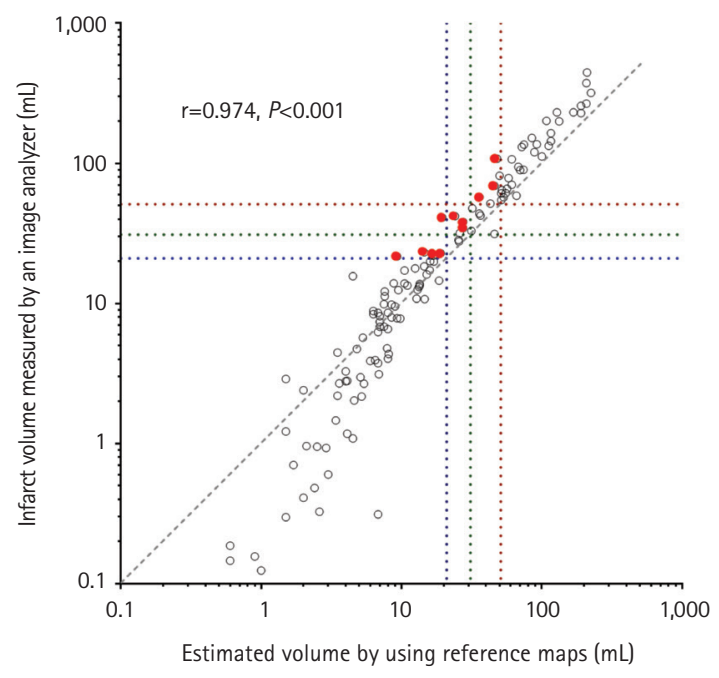

Supplementary Figure 7. A correlation plot of the infarct volumes estimated by a first-year resident using the reference maps vs. the infarct volumes measured by a vascular neurologist using an image analysis software package. Each dot indicates one patient's datum. Blue, green, and red dotted lines indicate infarct volume thresholds of 21,31, and $51 \mathrm{~mL}$. The colored dots indicate the data that were misclassified for the classification of volumes of $>21,>31$, and $>51 \mathrm{~mL}$. The gray dotted line indicates a perfect estimation slope $(x=y)$. Owing to a skewed distribution of infarct volumes, the $x$-axis and $y$-axis were logarithmically transformed for better visualization. 


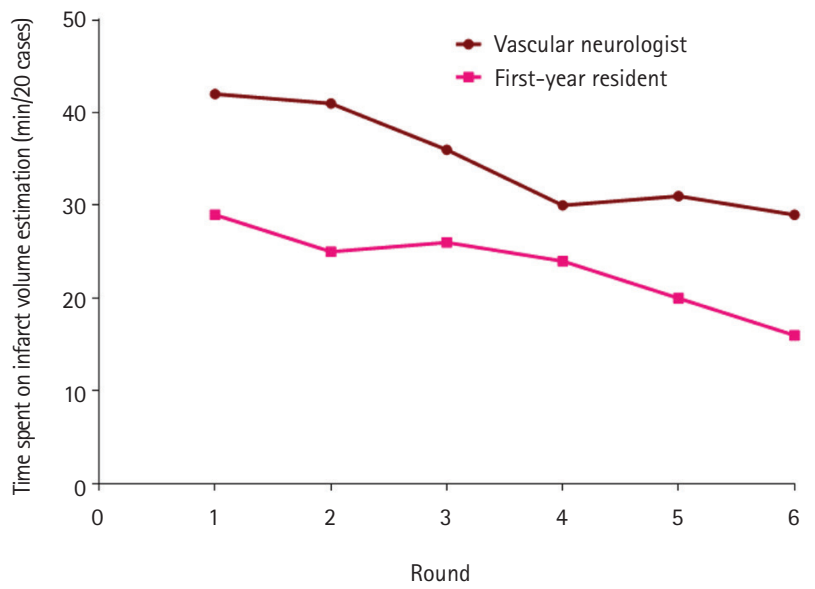

Supplementary Figure 8. Learning curve for the infarct volume estimation with the reference maps: vascular neurologist versus first-year resident. Each round is for 20 patients' diffusion-weighted magnetic resonance images (cases). 